\title{
A proteomic model of SARS-COV2 infection by comparing the interactomes of BRD4 with BET- inhibition and SARS-COV2 viral proteins - implications for re-purposing approved drugs or ubiquitin-mediated degradation of select candidates
}

GIRISH NALLUR ( $\square$ girish@vistarabio.com )

VISTARA BIOSCIENCE LLC

Research Article

Keywords: bromodomain, SARS-COV, Calnexin

Posted Date: April 23rd, 2020

DOI: https://doi.org/10.21203/rs.3.rs-24573/v1

License: (c) (i) This work is licensed under a Creative Commons Attribution 4.0 International License. Read Full License 


\section{Abstract}

The novel coronavirus SARS-CoV-2, the causative agent of COVID-19 respiratory disease, has infected $2,029,930$ people worldwide and caused 136,320 deaths. Consequently, the hunt for drugs showing efficacy against this deadly disease, or vaccines for prevention, are being intensely investigated. Unfortunately, there is a scarcity of research data on the molecular mechanisms of SARS-CoV-2 infection for quickly finding effective therapies, or repurposing existing drugs approved by the US FDA. This report models existing knowledge of SARS-COV2 viral proteins and the cellular proteins they interact with by comparisons with BRD4 interacting proteins identified from B cells, with or without BET inhibition. The E protein of SARS-COV2 interacts with BRD4, and the Spike (S) protein with CANX. Extensive similarities were observed with published cellular interactants of 13 SARS-COV2 proteins resulting in 47 BRD4interacting protein candidates, with or without BET inhibition. 61 cellular protein targets and 132 FDA approved drugs which use these proteins as targets are proposed, which can be investigated for efficacy against SARS-COV2 infections. The implications to SARS-COV2 disease diagnosis, therapy and vaccine creation are discussed.

\section{Introduction}

The recent outbreak of the COVID-19 pandemic has spurred intense research to understand the disease and identify biomarkers or drug targets. A detailed molecular phenotype of SARS- COV2 proteins and the cellular proteins they interact with can help efforts towards finding a cure. The RNA genome of SARSCOV-2 has 29,811 nucleotides, which codes for 28-29 proteins. Of these, the Spike, or S protein, is important for attaching to host cells, likely via the ACE-2 receptors, and injecting the viral RNA into the host cell for expression by the host cellular machinery. The synthesized viral proteins interact with cellular proteins to accomplish viral replication and repackaging. This report models potential impacts of virus and host protein interactions, since the cellular proteins control many signaling systems, including the immune system, the ubiquitination system, and bromodomain protein functions.

SARS-COV2 protein E has been reported to bind to bromodomain proteins in the host cells. Bromodomain 4 (BRD4) a BET sub-family protein, provides a recruitment platform by recognizing acetylated histones, associates with non-histone targets, and is also involved in NFKB signaling, Myc regulation, besides complexing with viral proteins. Additionally, BRD4 collaborates with other bromodomain proteins, some of which may also possess ubiquitination functions.

This report describes molecular phenotyping of BRD4 interacting proteins using Mass Spectroscopy of proteins from a human B cell line treated with BET inhibitors, compounds that bind to BRD4, and likely other bromodomain proteins. The BRD4 network proteins were modeled by comparisons with published data on SARS-COV2 interacting host proteins. The commonly identified proteins were mapped into pathways and complexes to model Interactome reprogramming in SARS-COV2 virus infected cells. These data will help with characterization of the SARS-COV2 infections, provide a model system for basic research, guide the selection of drug candidates, diagnostic reagents and vaccines for disease 
management, as well as serve panels of biomarkers to follow along the drug discovery process on a systems scale.

\section{Results}

BRD4 interacting proteins from human B cells. A pull-down assay using a bacterially encoded BRD4 partial protein (49-406 aa; BRD-pep) representing bromodomains I and II (BD- 1 and BD-II) as bait and proteins from DOHH2 cells as preys identified 884 distinct proteins with Mass Spectroscopy. Similar assays performed after treating the DOHH2 cells with JQ1, AZD5153, or I-BET151, yielded 1194, 1673, and 460 distinct proteins, respectively. Substantial similarities exist between the interactants identified across the 4 samples, yielding a total of 2,063 distinct proteins. These proteins, collectively designated the BRD4 network, were loaded into a database with the ability to separately track entries in each sample and to facilitate comparisons.

Bromodomain interacting proteins of SARS-COV2 commonly identified in BET inhibitor treated B cells. The database was queried for identities with the proteins interacting with the SARS-COV2 viral proteins, as reported by Gordon et al [1]. It is not known if DOHH2 human B cells are permissive for SARS-COV2 infection, but protein identities exist. As shown in Table l, 87 of the 330 SARS-COV2 interacting proteins reported by Gordon et al were also identified amongst the BRD4 network proteins. 24 of these interactions were mediated via NSP7 and ORF8 proteins of SARS-COV2. While only 8 of the 87 proteins complex directly with BRD4, 7 of these interaction similarities, including BRD2, occurred with the E, N, ORF8 or ORF10 proteins of SARS-COV2 (Fig. 1).. 18 other proteins from this set also interacted with these same viral proteins, suggesting that these proteins may be 'hubs' for interactions with host cellular proteins.

\section{ZC3H18 interaction with bromodomain proteins is unaffected by BET inhibition: Most SARS-COV2} interacting proteins selectively interacted with BRD4 depending upon the specificity of the BET inhibitor. $\mathrm{ZC} 3 \mathrm{H} 18$ was the only BRD4-complexing protein present in all 4 samples, and was not influenced by drug treatments (Table II).. Querying the database for proteins interacting with $\mathrm{ZC} 3 \mathrm{H} 18$ so as to extend the network, identified many proteins, including, CDC5L, PRPF4B, and FIP1L1 involved in splicing or RNA maturation, and RTF1, involved in the PAF complex aiding RNA polymerase II function and expression of WNT target genes (Table III), as BET inhibitors are known to do [4].. Importantly, RTF1 and NPM1 interact

with histones. Taken together, the absence of the histone regulator, RTF1, as well as of BRD2 and Histone H4C9 (see below) from the JQ1- or AZD5153-treated cells suggests that these compounds may dysregulate interactions of Histones with BRD4.

Histone interactions of bromodomain proteins in B cells: Histones exhibited characteristic patterns of interaction with any of 4 bromodomain proteins identified in the BRD4 network. In untreated DOHH2 cells, $\mathrm{H} 1, \mathrm{H} 3$, and $\mathrm{H} 4$ histones were identified [Table III], with H2AC4 being the only H2 histone. The histonebromodomain interactions varied with drug treatments of the cells, depending on the BET inhibitor used. BRD8 was recruited to BRD4-Histone complexes in I-BET151 treated cells, but it is not likely that it may have altered any Histone interactions, since the pattern was similar in untreated samples. Likewise, the 
effect of BRD3 identified in AZD5153-treated cells is not significant. BRD4 has been shown to interact with C-termini of H4 histones [5], and that such interaction is disrupted by JQ1. The major effect of the three compounds appears to be on $\mathrm{H} 4 \mathrm{C} 1$ and $\mathrm{H} 4 \mathrm{C} 9$, which is more pronounced in JQ-1-treated cells, compared with AZD5153 or I-BET151 treatments. H2AC4 was the only H2 histone observed in quiescent or DMSO treated DOHH2 cells, with most of the interactions involving $\mathrm{H} 1$ and $\mathrm{H} 4$ histones, consistent with the function of BRD4 as a nucleosome scaffolding agent binding to inter-nucleosomal regions.

In contrast, AZD5153 treatment showed markedly pronounced effects on $\mathrm{H} 2$ histones. Eight additional $\mathrm{H} 2$ Histones and BRD3 associated into the BRD4 network, as well as the linker histones, $\mathrm{H} 1-\mathrm{O}$ and $\mathrm{H} 1-$ 10. These included the variant Histones, $\mathrm{H} 2 \mathrm{AX}, \mathrm{H} 2 \mathrm{AZ2}$, and MACROH2A1, which are normally required for checkpoint-mediated arrest of cell cycle progression and chromosome segregation. These additional Histones interact with one another, as well as with many other proteins in the AZD5153-treated samples, including, BRD3, BRD4, TRIM28, PARP1, and proteins involved in chromosome maintenance, histone modification, and Transcription. Other potential candidates for the BRD2-mediated histone interactions in AZD- 5153-treated cells include, PURA and TERF2.

TRIM28 likely has diverse roles in B cells: TRIM28, another bromodomain containing protein possessing RING- and SUMO-type E3 ligase activities, was identified in all samples. TRIM28 interacts with all the other bromodomain proteins and Histones identified in this study.

Additionally, a large sub-network of TRIM28 interacting proteins was identified in this study (Table IV and Fig. 2.).. These included proteins interacting with Histones (PARP1, EP400, DLST, NPM1, NASP, NCL, SET, and SSRP1), proteins possessing ubiquitination roles, such as, RAD23B, SUMO2, CCDC50, and Ubiquillins, signaling and cell cycle proteins (IKBKG and CDC5L), and proteins involved in splicing, mRNA maturation, translation and transcription.

PARP1. A few proteins involved in ADP ribosylation of Histones were also identified in this study. Importantly, the Histone interactions of TRIM28 appear not to vary with BET inhibitor treatment, so it is likely that it plays a housekeeping role with bromodomain-histone interactions. Alternately, it may be involved in proteostasis or disassembly of the complexes.

SARS-COV2 viral protein E disrupts bromodomain:Histone interactions: Gordon et al (1) reported that the transmembrane protein E of SARS-COV2 virus binds to BRD2 and BRD4 by virtue of sequence homology with histone $2 \mathrm{~A}$, potentially disrupting BRD-histone binding by mimicking histone structure. In this report, six H2A histones were identified which associate with BRD4 in AZD5153-treated DOHH2 cells (Fig. 3a.).. Optimization of peptide signatures in the Mass Spectroscopy signatures for this sample suggest that the interactions may occur via the $\mathrm{N}$-termini of the Histone proteins (unpublished).

Gordon et al report that the region of $\mathrm{H} 2 \mathrm{~A}$ histone protein bears sequence homology with 15 amino acids of SARS-COV2 protein E (1).. As shown in Figure 3a, the $6 \mathrm{H} 2 \mathrm{~A}$ histones possessed the same canonical sequence homology with the SARS-COV2 E protein in their $\mathrm{N}$ - termini, suggesting that these could be 
targets for interaction with the E protein. The significance of this is not understood, and implications to SARS-COV2 infection will require an in-depth study of these interactions.

In B cells, all five histones and TRIM28 likely interact with PARP1, but the outcomes of such interaction is presently unknown. Moreover, PARP1 was identified in the BRD4 network in all samples employed in this study, as were many other common interactants of PARP1. Further characterization of the role of PARP1 in this network may help evaluate it as a target for therapy in SARS-COV2 infections, particularly in view of the involvement of ADP ribosylations in SARS- COV2 biology. Also, characterization of many other proteins identified in this subnetwork of PARP1 and Histone H2A proteins, involved in histone recognition/modification, chromosome maintenance, transcription and ubiquitination may help identify a drug candidate via small or large molecule or proteostasis therapy.

Modeling Histone H2 disruptions in SARS-COV2 infection and BET inhibitor-treated B cells: Gordon et al report that the SARS-COV2 protein E mimics the histone to disrupt its interaction with BRD2, thus inducing changes in host's protein expression that are beneficial to the virus. However, it is not clear as to how many $\mathrm{H} 2 \mathrm{~A}$ histones were identified in their study, or which homologs. Of the nine $\mathrm{H} 2$ histone proteins identified in this study, only 6 homologues possessed the signature sequence homologous with the SARS-COV2 E protein. Of these, only H2AC4 was associated with a BRD4 network in untreated B cells, the remaining were all specific to AZD5153 treatment. This suggests the intriguing possibility of modulating the AZD5153-responsive set of six H2A histones with AZD5153 or an analog to at least manage outcomes in SARS-COV2 infection. For instance, recruitment of these H2A histones could increase the threshold for the viral E protein and alleviate some disruption of bromodomain- histone interactions, such as by squelching.

Nevertheless, the available information allows a simplistic model to assess outcomes in SARS- COV2 infections relating to dysregulation of Histone-bromodomain protein interactions (Fig. 3b).. BRD4, BRD2 and TRIM28 appear to be the minimal bromodomain protein requirements for interaction with Histones in normal B cells. Treatment with BET inhibitors disrupts BRD4 function, and may have additional effects by recruiting BRD2, BRD3 or BRD8. As shown in this report, many Histone interactions are altered in these cells with BET inhibition, and likely have additional impacts on transcription, chromosome maintenance or related pathways operating in normal cells. Likewise, in SARS-COV2 infected cells, binding of the $E$ protein likely alters BRD2 interaction with some $\mathrm{H} 2$ histones, thereby disrupting the histone code. It is not known if any bromodomain protein other than BRD2 and BRD4 was identified in the study by Gordon et al, or any other histone chaperones, histone modification proteins and HDACs, since these have been identified in this study. Yet, the net outcomes with BET inhibition or SARS-COV2 infection could be the same - dysregulated transcription and/or chromosome maintenance. Replicating this study with the appropriate samples with recombinant SARS-COV2 proteins or virus infected cells is needed to more clearly understand the underlying mechanisms. For now, the model proposes that disruption of bromodomain functions could be one way that the SARS-COV2 virus takes control of the host cells. 
Calnexin (CANX) may be important for infectivity of SARS-COV2: Fukushi et al [6], identified CANX as the cellular partner of SARS-COV Spike protein (S protein). They suggested that the $\mathrm{N}$-terminal $\mathrm{S} 2$ domain (S2-N) of S protein was important for SARS-COV infection. They also noted that homologues of CANX, namely CALR and PDIA3/ERp57, associate to prevent premature degradation of nascent polypeptide chains and help manage folding of the $S$ protein. This interaction was inhibited by a-glucosidase inhibitors.

In this study, CANX was identified as an interactant in the BRD4 network, in JQ1 or AZD5153- treated cells. CALR and PDIA3 were present in all samples. A 122-protein signature of CANX interacting proteins was also identified in each of JQ1 and AZD5153 treated cells (Fig. 4)..

Included in this set are, NUP98, which binds to ORF6 of SARS-COV2 virus, and NUP153, involved in assembly of the nuclear pore complex (NPC) and facilitating transcription. TPR, a third component of the NPC was also identified in both samples, and DHX9, a helicase which facilitates transcription in collaboration with NUP98 was identified in AZD5153-treated samples. NUP proteins complex with one another and with other proteins to comprise the NPC. These findings show further similarities with protein networks important for both BRD4 function and SARS-COV2 virus infection.

Interacting proteins for 9 SARS-COV2 viral proteins were also co-identified with the CANX subnetwork in $B E T$ inhibited $B$ cells: Overall, 17 CANX-interacting proteins were the same in the SARS-COV2 interacting proteins reported by Gordon et al. Included in this set were 4 additional NPC components, NUP50, NUP62, NUP210, and NUP214. 4 of the 6 NUP proteins interact with 3 SARS-COV2 virus proteins. The remaining 13 CANX-interacting proteins interact with 6 SARS-COV2 viral proteins. The similarities suggest that CANX and its interacting proteins, may be an important determinant of SARS-COV2 infection. Curiously, CANX was not present amongst the set reported by Gordon et al. Perhaps, cell type differences account for this.

SARS-COV2 may interact with the immune system via the CANX network: With JQ1- or AZD5153 treatments, however, many HLA antigens, including HLA-B, and beta-2 microglobulin, and CD74 were identified (Fig. 5).. While BRD4 is not known to interact with HLA-B nor CD74, the interactions may be mediated via beta-catenin, which was also identified in these samples or many other common interactants in the 122 protein CANX subnetwork in JQ1 and AZD5153 treated samples. All the HLA antigens identified, CD74 and B2M interact with CANX, and the signature included proteins essential for immune response, antigen presentation, innate immunity, and regulation of $\mathrm{T}$ cell function. Moreover, B2M primarily displays viral antigens, being implicated in a number of viral infections, such as, HIV, HCV, and EBV. The JQ1- and AZD5153-treated samples include many other proteins involved in antigen presentation.

The bromodomain-CANX axis may represent common mechanisms of viral pathogenesis and immune suppression by BET inhibitors: Lee et at [7] reported that mice dosed with JQ1 at efficacious exposures demonstrated on-target and dose-dependent decreases in their lymphoid and immune cell compartments. These were accompanied by a decrease in both B- and T-lymphocytes as well as peripheral white blood 
cells. In addition, there are numerous reports in the literature of BET proteins, mainly BRD4, interacting with viral proteins, and being involved in viral life cycles [2, and references therein]. Georgiev et al [3] found that MK-8628, a potent and highly selective inhibitor of BET proteins, has potent immunosuppressive effects on human T cell biology which correlated with cyclin-dependent reduction in expression of transcripts required for proper T lymphocyte function as well as suppression of polyfunctional cytokine production [3].. Their studies provide strong impetus for assessment of BET inhibitors in autoimmune $\mathrm{T}$ cell-driven inflammation. BET proteins have also been reported to have a role in regulating immune checkpoints, likely via interaction with PD1 [8].. Molecular mechanisms of immune suppression by BET inhibitors need to be elucidated, but may involve a complex interplay between proteins controlling B and T cell functions, the MHC, CD antigens, PDCD family members and other immunological checkpoint control proteins (unpublished observations).

CD74, a CANX interactant, collaborates with many $C D$ antigens involved in immune regulation in $B$ cells: $\mathrm{CD}$ antigens participate in immune reactions as receptors for cell communication (e.g. adherence molecules, antigen recognizing receptors). The BRD4-pep network contained signatures involving a total of $16 \mathrm{CD}$ antigens involved in immune response and maintenance, antigen presentation, and interactions with the $\mathrm{MHC}$ molecules, antigen presentation, $\mathrm{B}$ cell receptor signaling, $\mathrm{T}$ cell maturation, and cell-cell contacts (Table $V$ ).. CD74 may be represent a point of entry into, and a key contributor, for the signals to be transmitted from the SPIKE protein of SARS-COV2 through to the immune system components, via CANX. Incidentally, CD74 binds to the cytokine MIF, a pro-inflammatory cytokine involved in the innate immune response towards bacterial pathogens and regulation of macrophage function. MIF was identified in JQ1- and AZD5153-treated samples. CD36 was the only CD molecule in this set whose interaction with the BRD4 network was lost in all samples with BET inhibition, and may be another key contributor. More studies in appropriate cells are needed to test the likelihood, and elucidate the mechanisms with pure proteins.

BET inhibition may disrupt MAPK1 interaction with the BRD4 network: A total of 206 proteins lost interaction with the BRD4 network when cells were treated with any of the BET inhibitors. 7 of these proteins interact with 5 SARS-COV2 encoded proteins (Table VI).. IF the same interactions also occur in virus infected cells, it implicates 2 new viral proteins not hitherto identified as interacting with proteins in the BRD4 complex, ORF3A and ORF 9C, bringing the number of SARS-COV2 viral proteins implicated to 12. MAPK1 is a key protein whose interaction with BRD4 is lost in BET-inhibited B cells, which plays an important role in the MAPK/ERK cascade (Fig. 6).. Many other proteins complexing with MAPK1 were also lost, with JQ1 and AZD5153 treatments showing the most pronounced effects. CANX is a known target for phosphorylation by MAPK1, so it is not clear if the loss of MAPK1 interaction in BET inhibited cells may also regulate CANX function. Since MAPK1 requires activation by other kinases acting upstream, this dataset needs to be queried further to identify them. Nevertheless, these findings suggest that MAPK1 may be a point of interaction of viral proteins in SARS-COV2 infection. Further studies of this nature may confirm the hypothesis. 
Modeling signaling mechanisms in SARS-COV2 infection: A model is presented which takes into account the aforementioned protein contacts in BET inhibited B cells and their similarities with SARS-COV2 virus interacting proteins. This model includes 47 cellular proteins and 13 SARS-COV2 proteins. While complex formation is expected amongst many of the cellular proteins interacting directly with the SARS-COV2 proteins, many other cellular proteins identified in this study interact with both BRD4 and CANX, and may serve to pass signals across. A full analysis of all such hub proteins identified in this study was not possible, only a few are mentioned, including, FYN, OS9, ITGB1, CTNNB1, and MAPK1. Replicating these data in SARS-COV2 appropriate cells with virus infection or recombinant viral protein expression will help further characterize the signaling mechanisms. Doing so will not only help characterize molecular interactions in SARS-COV2 infections, but may yield valuable clues for the underlying reasons of mortality and morbidity with these infections. These studies may help validate how basic cellular systems, such as, Chromosome maintenance, Transcription, immune response, or susceptibility to SARSCOV2 infections manifest in the population, and pave the way to finding cures and diagnosis of SARScov2 infections.

Repurposing existing drugs to treat SARS-COV2 infections: Based on the BRD4 networking proteins and their similarities with SARS-C)V2 viral protein associating host proteins, a list of potential targets and the FDA-approved drugs for the target is proposed (Table VII). CDC5L, CANX, bromodomain proteins, CDK12, MAPK1, or other kinase or phosphatase inhibitors may be strong candidates. Whether these drugs provide benefit in treating SARS- COV2 infections remains to be seen.

\section{Conclusions}

This report describes key findings of the BRD4 interactome under conditions involving BET inhibition of DOHH2 cells, a human diffuse large B cell lymphoma cell line. Cells were treated with JQ1, AZD5153, or IBET151, protein preparations therefrom were used for pull-down assays, and the BRD4 interactomes were identified with Mass Spectroscopy. While many changes to the interactome were detected, in general, JQ1 and AZD5153 BRD4 networks showed a similar pattern, in contrast to I-BET151, which more closely resembled DMSO controls, despite showing many other changes.

Our data were modeled by comparisons with published information about the SARS-COV2 proteins and their interaction with cellular proteins. This analysis helps develop a level of understanding of the key components, contact points and pathways of infection, which after replication in appropriate cell systems, may guide the development of diagnosis or treatments. 61 cellular proteins bearing identical interaction signatures with 13 SARS-COV2 proteins correlate with the BRD4 database components. These originate from either the STRINGseeker (Interactomics) or UBQuest (Ubiquitin fingerprinting) platforms, and are being separately tracked. Work is ongoing to query the database for additional proteins collaborating with these initial set of candidate proteins and map them into either signaling or ubiquitinmediated degradation pathways. 
The findings in this report have flagged many signaling mechanisms emanating from cellular receptors, the $\mathrm{MHC}$, immune response, histone readers, chromosome maintenance, transcription, cell-cell contacts, nuclear pore organization and transport across membranes, as well as RNA maturation, splicing and translation. These markers will serve as starting points for translational studies, and use the results therefrom to query these data for additional expansions to the networks. Many potential candidates for drug interventions have been identified and can be expanded with more translational information. Knowledge of more cellular proteins interacting with each of the SARS-COV2 proteins can help with a holistic view of the SARS-COV2 disease process. Nevertheless, the comparison model proposed here extends current knowledge and paves the way for translational studies, besides adding perspectives on the involvement of CANX and the pathways involving the protein.

Other than the mortality and morbidity of SARS-COV2 infections, a prime concern is that the virus may compromise immunity in infected individuals and pose long term effects on the health of affected patients. Identifying markers or signatures of immune responses in SARS-COV2 infections can help develop molecular tests as well as guideposts for creating better and safer vaccines. SARS-COV2 proteins interacting with the CANX subnetwork may need to be investigated in greater detail since this seems like a possible route for the virus to gain entry into the immune system in some manner. More studies are needed to identify the full complement of proteins and possible other types of molecules which comprise these sub-networks.

An initial set of drugs approved by the US FDA is proposed, based on the network information, and proximity to the SARS-COV2 protein via complex formation. Many other candidates likely exist for creating for new drugs from and can be identified with more in-depth probing of the BRD4 network, which are amenable to small molecule, large molecule or peptide drugs.

\section{Methods}

Cells and reagents: DOHH2 were purchased from Creative Biomart) and SU-DHL-4 (CRL- 2957), SU-DHL6 (CRL-2959), Z138 (CRL-3001) and Z138 (CRL-2632) cell lines were from ATCC. Cells were maintained in culture according to manufacturers' recommended protocols. JQ1 (S7110) and AZD5153 (S8344) were purchased from SelleckChem. Pierce antibody biotinylation Kit (90407) was purchased from ThermoFisher.

Pull down assays: 2 ug bait proteins were spotted onto PROTRAN nitrocellulose filter discs (Schleicher \& Schuell) equilibrated in PBS and let to stand at room temperature for $15 \mathrm{~min}$. Spotted filters were rinsed in PBS and blocked for $4 \mathrm{hr}$ with 3\% BSA in PBS. After washing with 3 changes of PBS for 5 min each with shaking, the filters were incubated overnight with 100 ug of total proteins at room temperature with gentle agitation. Filters were washed with 3 changes of PBS for 5 min each with agitation and the bound proteins collected in PBS containing 0.1\% SDS. Mass spectrometry analysis was performed by the Biological Mass Spectrometry Facility of Robert Wood Johnson Medical School and Rutgers, The State University of New Jersey (http://cabm-ms.cabm.rutgers.edu/). Mascot software was used to identify 
proteins from LC-MS/MS data against the Swiss-Prot human database using a $1 \%$ false positive discovery rate (FDR).

Whole cell lysate preparation: $4 \times 106$ cells were resuspended in 400 ul of $1 \times$ Cell Lysis buffer $(9803$, Cell Signaling Technology) and incubated at room temperature for $15 \mathrm{~min}$ with occasional mixing. Lysates were cleared by centrifugation and the supernatants stored in aliquots at $-20^{\circ} \mathrm{C}$.

Drug treatments, Cell viability assay and combination screening: Cells were grown for 72 hours at $37^{\circ} \mathrm{C}$ under $5 \% \mathrm{CO}$. Cell viability was measured by staining cells with Trypan Blue and counting in a hemocytometer. For drug treatments, cell suspensions ( $100 \mu \mathrm{l}$ per well) were seeded into 96-well UBottom plates (351177, Corning) and increasing concentrations of compounds were added to the wells in a $6 \times 6$ dosing matrix. After 3 days of growth, proliferation was measured using CellTiter 96 AQueous One Solution Cell Proliferation Assay (G3582, Promega) according to the manufacturer's protocol. Twodimensional dose response matrix and curve fitting were performed according to Chou-Talalay3. Experiments were performed in triplicate.

Data analysis: Protein interactions analysis and networks modeling were performed using the information from CORUM (https://mips.helmholtz-muenchen.de/corum/),, GeneCards (https://www.genecards.org/Search/Keyword?queryString = HSP90B1),, and BioGRID (https://thebiogrid.org/) databases.

\section{References}

1. Gordon et al. A SARS-CoV-2-Human Protein-Protein Interaction Map Reveals Drug Targets and Potential Drug-Repurposing. doi: https://doi.org/10.1101/2020.03.22.002386.

2. Stathis A and Bertoni F. BET Proteins as Targets for Anticancer Treatment. Cancer DOI: 10.1158/2159-8290.CD-17-0605.

3. Georgiev at al. BET bromodomain inhibition suppresses human T cell function. Horiz. 3(7): 294-305. 2019.

4. Engelke, C.G. and Chinnaiyan, A.M. aBETting therapeutic resistance by Wnt Cell Res. 25, 1187-1188 (2015).

5. Jung et al. Affinity map of bromodomain protein 4 (BRD4) interactions with the histone $\mathrm{H} 4$ tail and the small molecule inhibitor JQ1. J Biol Chem. 89(13): 9304-19. (2014).

6. Fukushi et al. Monitoring of S Protein Maturation in the Endoplasmic Reticulum by Calnexin Is Important for the Infectivity of Severe Acute Respiratory Syndrome Coronavirus. Virol. 86(21): 1174511753. (2012).

7. Lee et al. Nonselective inhibition of the epigenetic transcriptional regulator BET induces marked lymphoid and hematopoietic toxicity in mice. Toxicology and Applied Pharmacolog 300(1) 47-54. (2016).

8. Doroshow et al. BET inhibitors: a novel epigenetic approach. Oncol. 28: 1776- 1787. (2017). 


\section{Tables}

Due to technical limitations, Table 1-7 are provided in the Supplementary Files section.

\section{Figures}

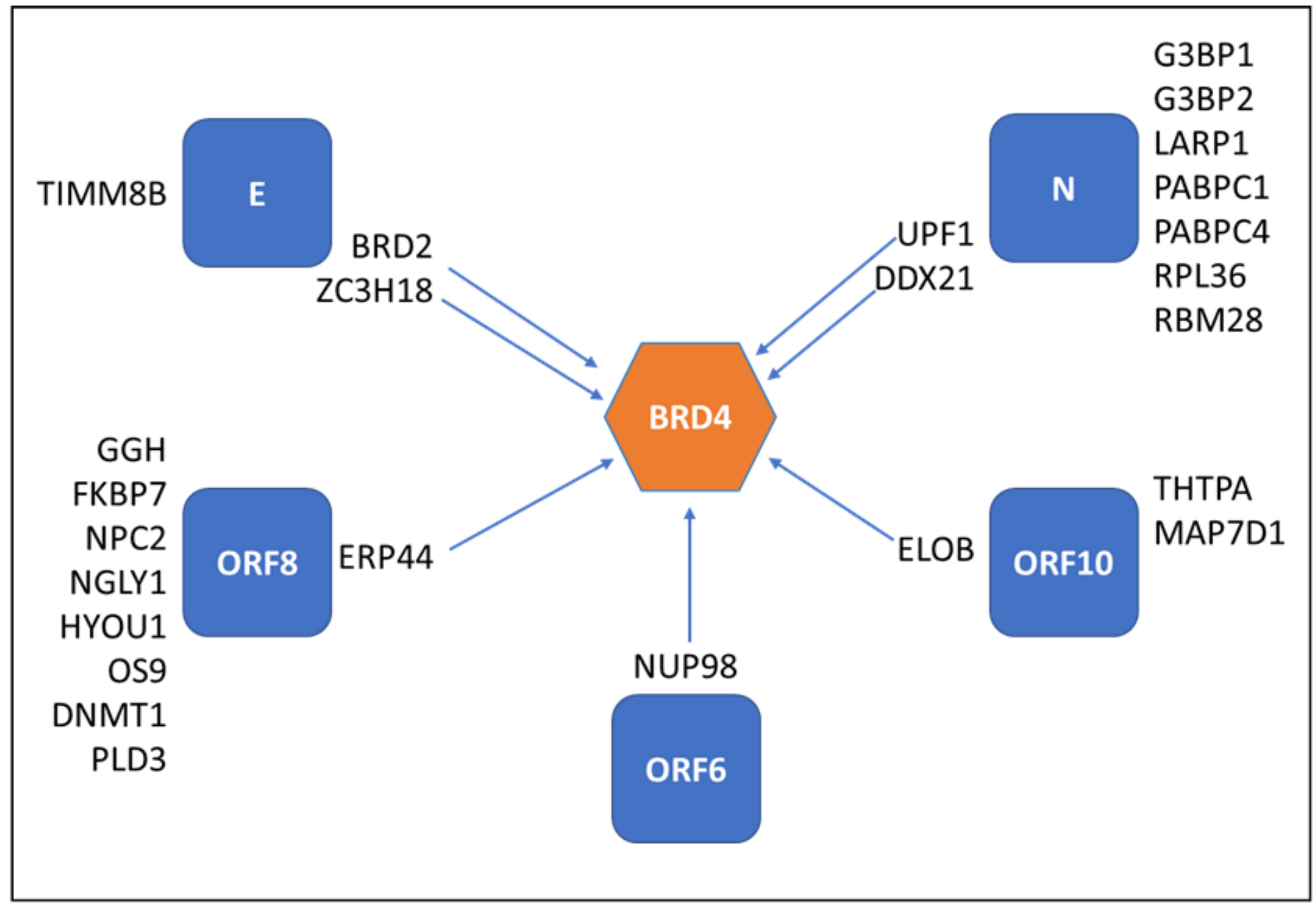

Figure 1

8 proteins complexing with 5 SARS-COV2 proteins interact directly with BRD4-pep. 


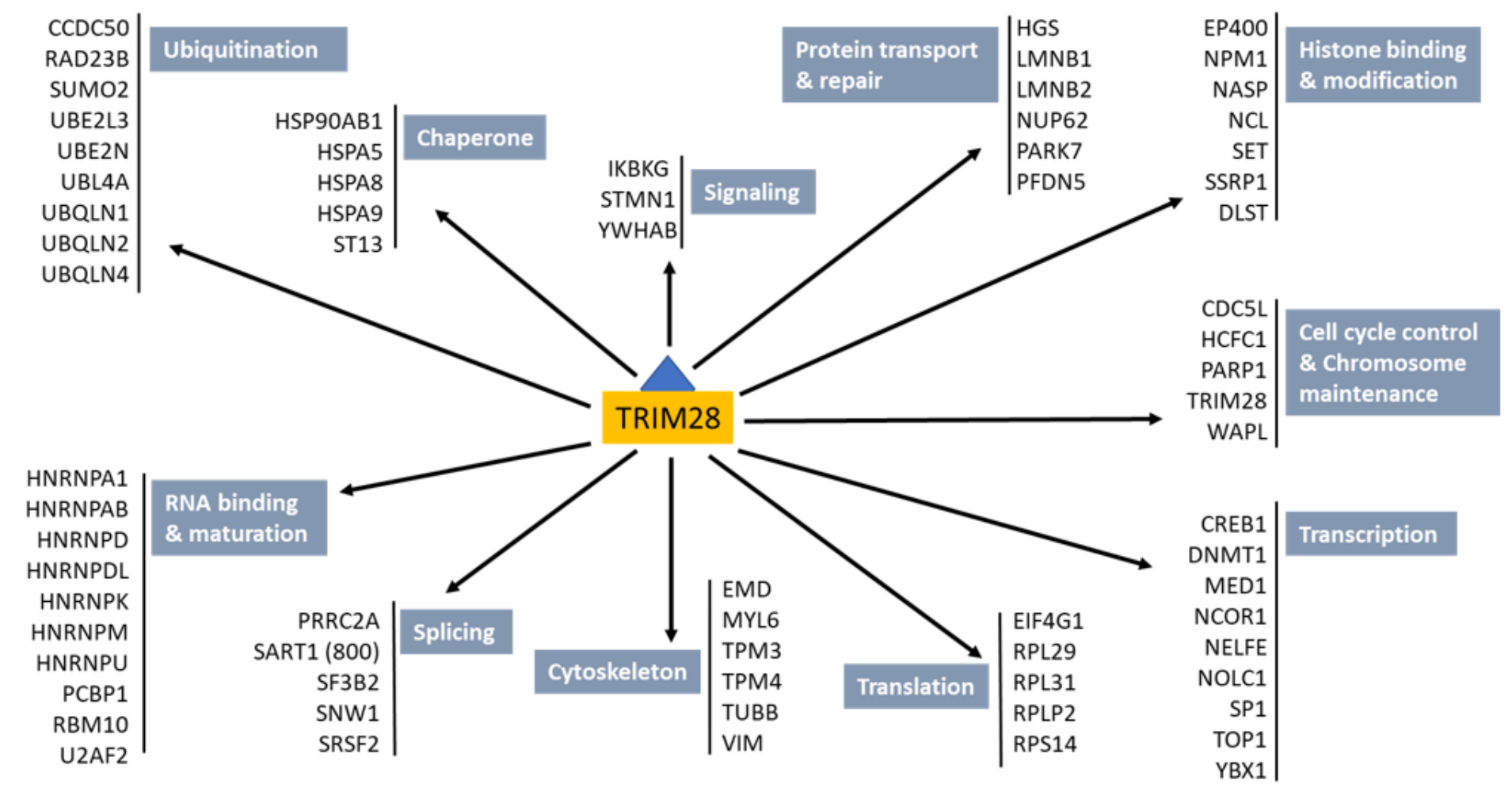

Figure 2

Selected interactions of TRIM28 in BET inhibitor-treated B cells. 
a. Homology of identified H2A proteins with the E protein of SARS-COV2

\begin{tabular}{r|r|r|}
\cline { 2 - 3 } E /SARS-COV2 & \multicolumn{2}{|c|}{ VFLLVTLAILTAXXLLAAYAANIVNVSLVKPTVYVYSRVKNL } \\
\cline { 2 - 4 } H2AZ2 & P16104 & aervgagapvylaavleyltaeilelagnaardnkktriiprhlqlairndeelnkllgg \\
\cline { 2 - 4 } H2AC4 & P04908 & gnyservgagapvylaavleyltaeilelagnaardnkktrii \\
\cline { 2 - 4 } H2AC12 & Q8N257 & aervgagapvylaavleyltaeilelagnaardnkktriiprhlqlairn \\
\cline { 2 - 4 } H2AC20 & Q16777 & aervgagapvymaavleyltaeilelagnaardnkktrii \\
\cline { 2 - 4 } MACROH2A1 & O75367 & hpkyrigvgapvymaavleyltaeilelagnaardnkkgrvtprh \\
\cline { 2 - 3 } & &
\end{tabular}

b. Proposed model of dysregulated Histone : BRDx interactions

Normal B cells

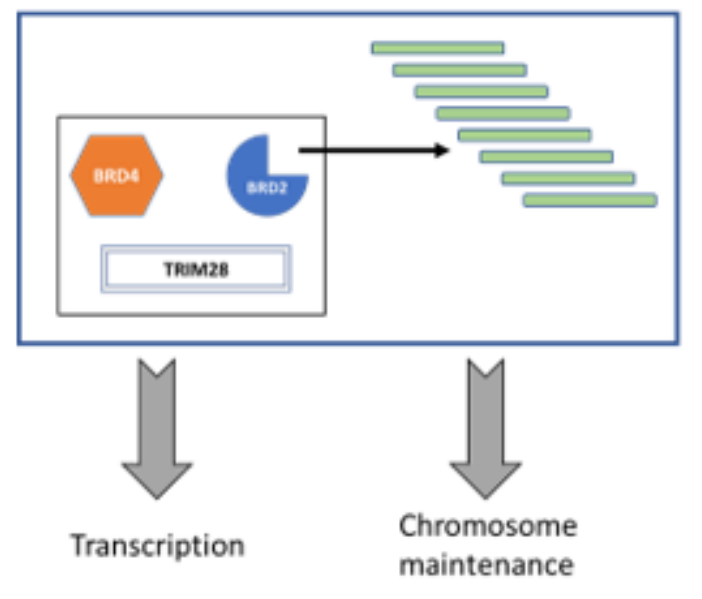

\section{B cells with BET inhibition}

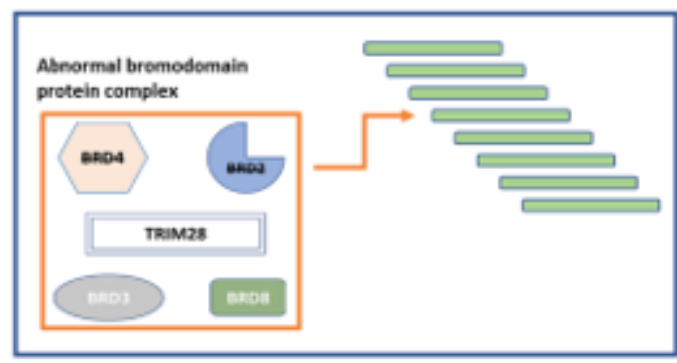

\section{SARS-COV2 infected cells}

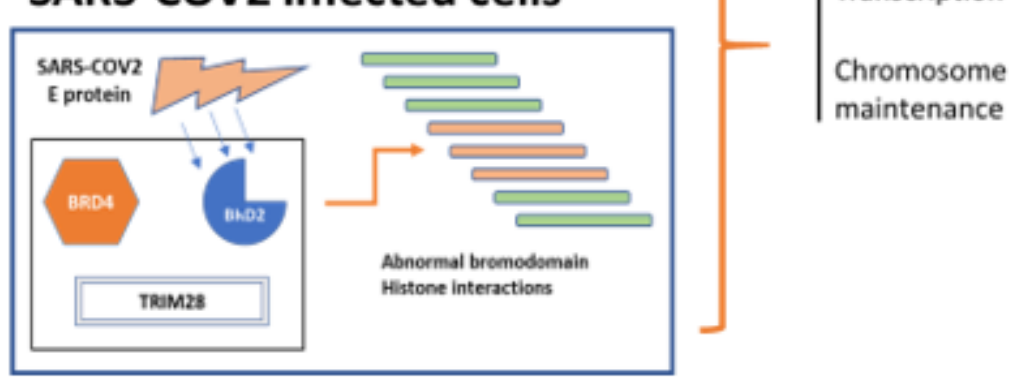

Figure 3

Impairment of bromodomain function is common to BET inhibition in B cells and SARS- COV2 E protein mediated effects. 


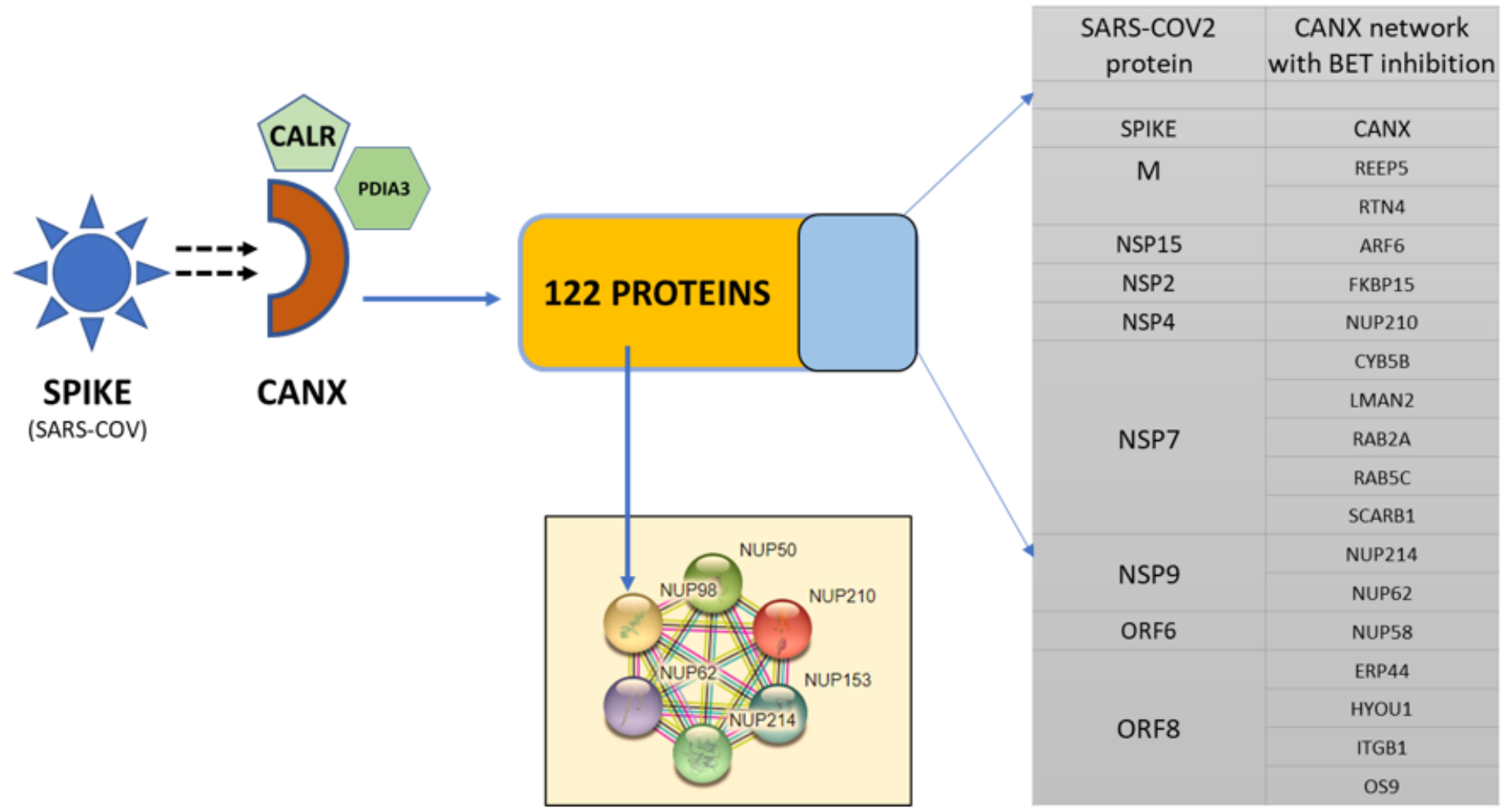

\section{Figure 4}

Calnexin (CANX) and its interactants in BET inhibited cells share identities with 9 SARS- COV2 interacting cellular proteins.

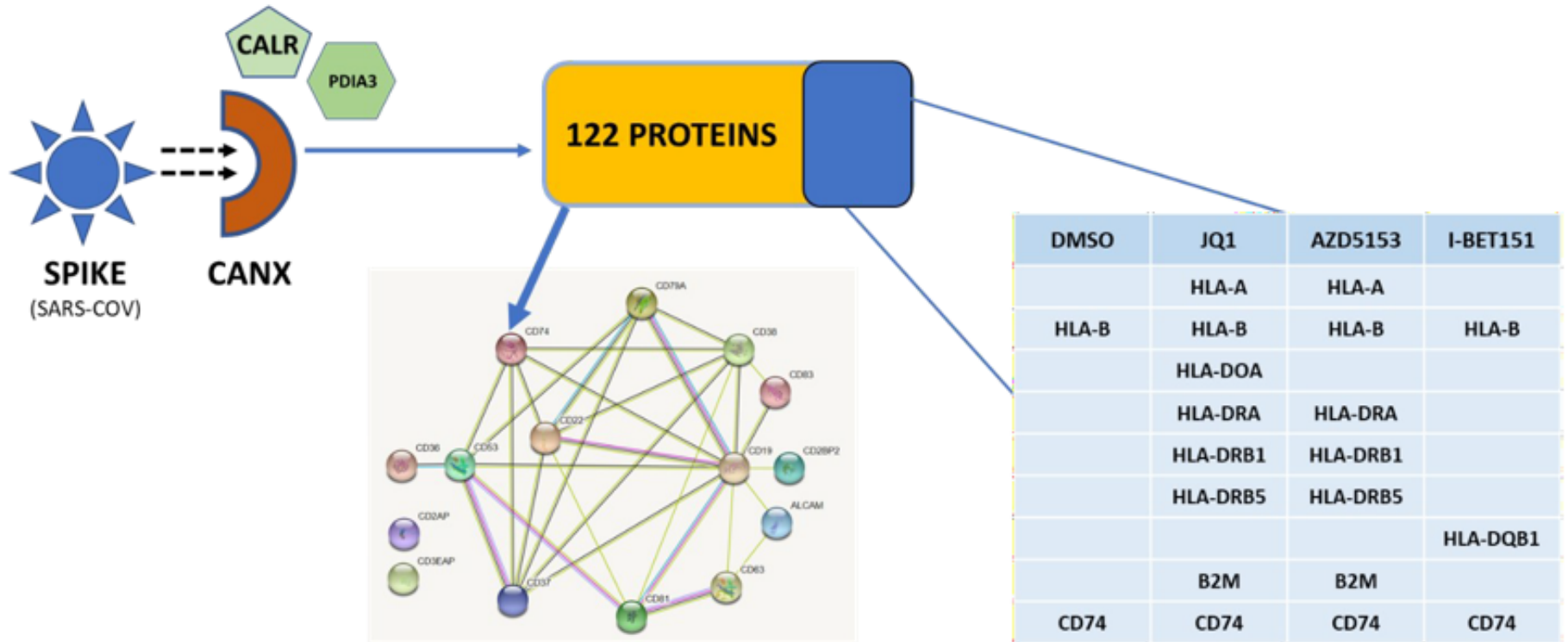

Figure 5 
SARS-COV2 S protein may interact with the immune system via CANX interacting proteins.

\begin{tabular}{|c|c|c|c|c|}
\hline PROTEIN & DOHH2 & JQ1 & AZD5153 & I-BET151 \\
\hline MAPK1 & $\nabla$ & & & \\
\hline MAPKIP1L & & $\nabla$ & $\nabla$ & \\
\hline ARHGEF2 & & & $\nabla$ & \\
\hline BAD & & & $\nabla$ & \\
\hline CANX & & $\nabla$ & $\nabla$ & \\
\hline CDK2AP1 & & $\nabla$ & $\nabla$ & \\
\hline ERF1 & & & $\nabla$ & \\
\hline MAP2 & $\nabla$ & $\nabla$ & $\nabla$ & $\nabla$ \\
\hline PIN1 & $\nabla$ & $\nabla$ & $\nabla$ & $\nabla$ \\
\hline STMN1 & $\nabla$ & $\nabla$ & $\nabla$ & $\nabla$ \\
\hline
\end{tabular}

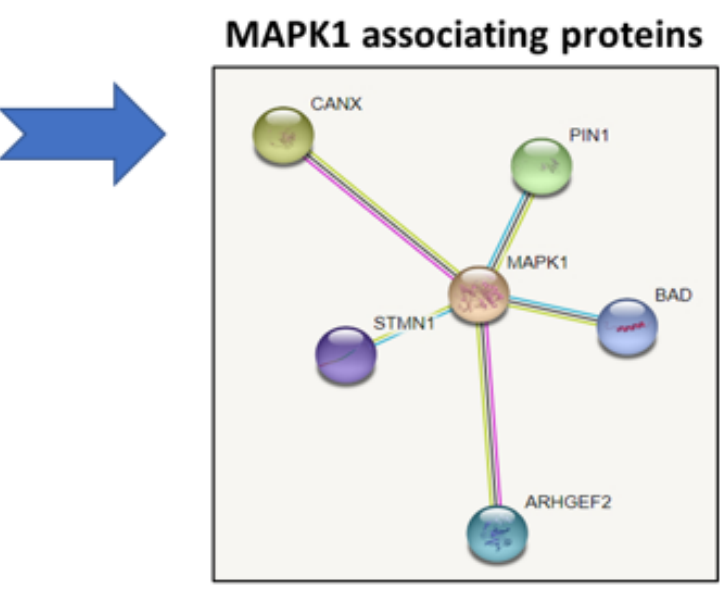

\section{Figure 6}

BRD4 interaction with MAPK1 is lost in BET inhibited cells.

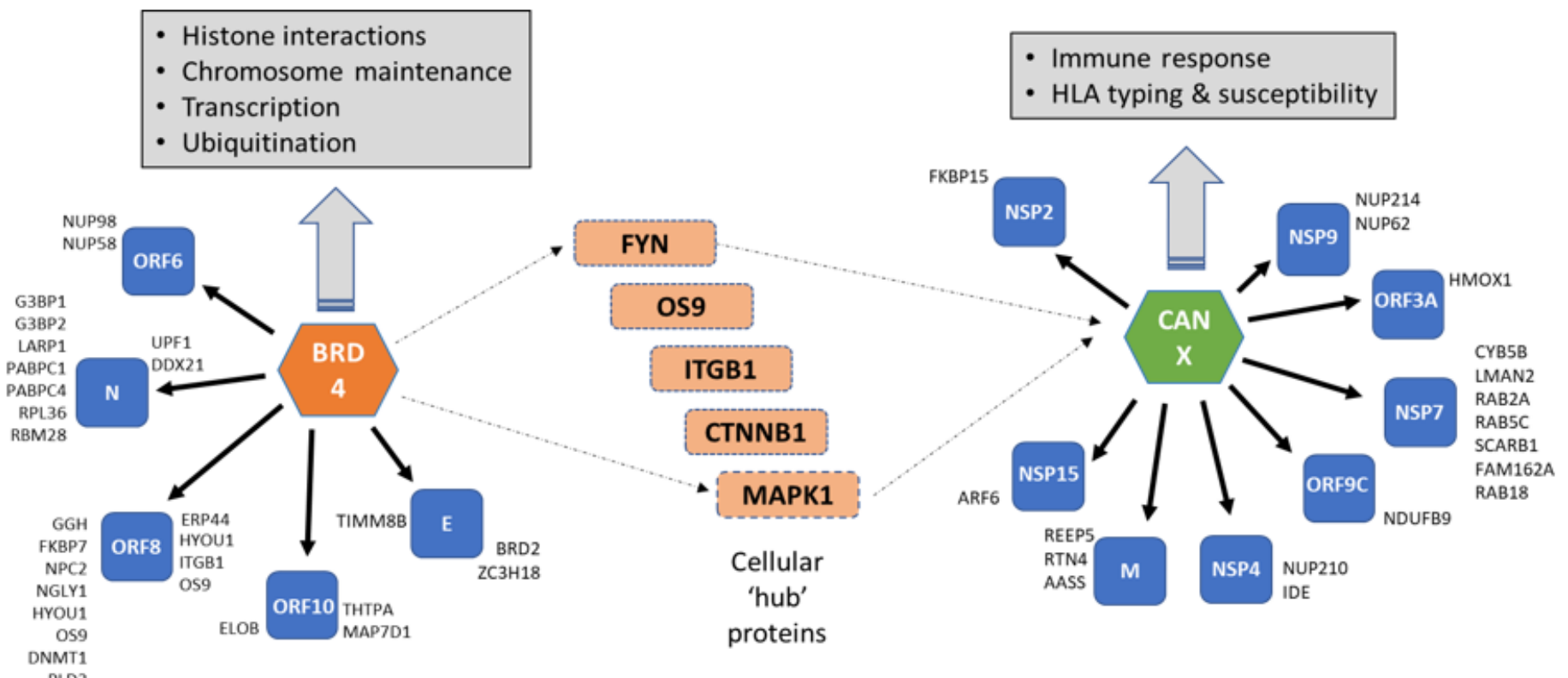

Figure 7

An interactions model of 13 SARS-COV2 proteins with proteins in the BRD4 network in B cells. 


\section{Supplementary Files}

This is a list of supplementary files associated with this preprint. Click to download.

- Tables.docx 